\title{
Provider Attitudes toward the Use of Hepatitis C Virus-Positive Organs in Kidney Transplantation
}

\author{
Thomas Couri $^{a}$ Joshua Katz $^{\mathrm{a}}$ Kate Stoeckle $^{\mathrm{b}}$ Aishwarya Nugooru ${ }^{\mathrm{c}}$ Heidi Yeh ${ }^{\mathrm{d}}$ \\ Raymond Chung ${ }^{\text {e Sonali Paul }}{ }^{f}$

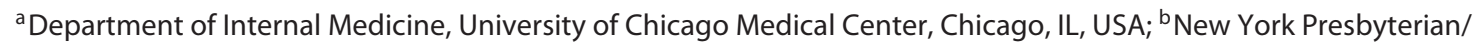 \\ Weill Cornell Medical Center, Department of Internal Medicine, New York, NY, USA; ${ }^{\circ}$ Virginia Commonwealth University \\ Medical School, Richmond, VA, USA; d Department of Surgery, Section of Transplant Surgery, Massachusetts General

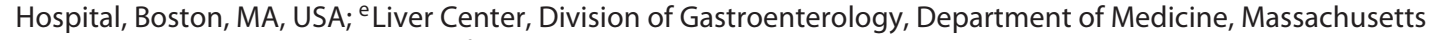

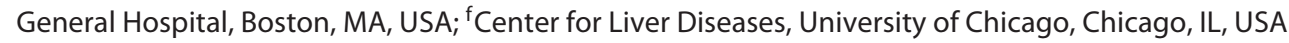

\section{Keywords}

Hepatitis C . Organ allocation - Kidney transplantation ·

Provider education

\begin{abstract}
Background: Direct-acting antivirals have changed the landscape of hepatitis C virus (HCV) care. While transplantation with HCV-positive donor organs is increasing, little is known about providers' attitudes toward this topic. The aim of this study is to determine providers' attitudes toward HCV-positive kidney transplantation. Methods: Willing transplant and nontransplant nephrologists, transplant surgeons, and mid-level providers completed an online survey from April through May 2018. The survey asked about HCV knowledge and willingness to transplant HCV-positive antibody, nucleic acid testing-positive kidneys into HCV-negative recipients. Descriptive analyses including mean and median for continuous variables and frequencies for categorical variables were calculated. Results: Seven-hundred surveys were emailed and 99 providers (62 transplant nephrologists, 28 nontransplant nephrologists, 7 transplant surgeons, and
\end{abstract}

\section{KARGER}

(c) 2019 S. Karger AG, Basel

E-Mail karger@karger.com

www.karger.com/ajn
2 advanced practice providers) completed the survey (participation rate $14.1 \%$ ). All providers knew that HCV was curable, with $60 \%$ believing that it had no effect on transplant success and $32 \%$ thinking it reduced transplant success. Providers were significantly more likely to offer a HCV-positive organ to HCV-positive recipients compared to HCV-negative recipients in all queried circumstances ( $p<0.005$ in all cases), especially with increasing impact on patient's quality of life. While only $39 \%$ of providers would offer a HCV-positive organ for transplant to a patient without $\mathrm{HCV}$ if it reduced the waitlist time by 1 year, $92 \%$ would offer a HCV-positive organ if it reduced the waitlist time by 4 years. However, only $47 \%$ thought that the use of HCV-positive kidneys should be for routine care, while $38 \%$ believed it should be reserved for research purposes only. There were no significant differences between transplant and nontransplant nephrologists in attitudes toward HCV-positive kidney transplantation. Providers believed that donor organs from those who were obese, $>50$ years old, or had died from a cardiac arrest were

T.C. and J.K. co-first authors. 
significantly more likely to reduce the likelihood of a successful transplant 1-year posttransplant when compared with a HCV-positive organ ( $p<0.005$ in all cases). Eighty-six percent of providers had concerns about HCV curability posttransplant. Conclusion: Although 92\% of providers were willing to offer a HCV-positive kidney for transplant as patient waitlist time increases, less than half supported offering HCVpositive transplantation for routine care rather than for research. The results underscore the need for further education and data about the efficacy and safety of HCV-positive kidney transplantation.

(c) 2019 S. Karger AG, Basel

\section{Introduction}

Donor organ scarcity remains a significant issue facing transplant medicine today. In the last 30 years, the kidney transplant (KT) waitlist size has increased by $600 \%$ in the absence of a corresponding increase in donations [1]. According to the 2016 Organ Procurement Transplant Network/Scientific Registry of Transplant Recipients annual report on KT, 30,869 adult patients were newly added to the transplant list in 2016. While inequities exist in KT rates across donation service areas, as little as $9.1 \%$ of KT candidates are transplanted within 5 years. Mortality rates for KT candidates on the waitlist range from 0 to 2.7 per 100,000 patient-years [2].

To improve donor graft shortages, hepatitis $\mathrm{C}$ virus (HCV)-positive donor grafts are being used across all solid organ transplantation [3]. In the direct-acting antiviral (DAA) era, HCV treatments are well tolerated, and most regimens have nearly $100 \%$ cure rates. HCV positivity affects $1-2 \%$ of the U.S. population, an estimated $1.8-8 \%$ of KT recipients, and $4-18 \%$ of potential organ donors [35]. Prior to the DAA era in $2014, \mathrm{HCV}$ cure rates using interferon-based regimens were approximately $50 \%$ and even lower (18-34\%) for KT recipients [6, 7]. In addition, in the pre-DAA era, HCV-positive kidneys were often not transplanted due to the near certainty of HCV transmission during transplant, as well as increased risk of mortality and graft loss in HCV-positive patients after KT. Between 2005 and 2014, 4,144 HCV antibody-positive kidneys, most of which were of good quality, were discarded [8]. These kidneys, however, may have not had active $\mathrm{HCV}$ and were only antibody positive as nucleic acid testing (NAT) status, a more accurate marker of active infection, was not routinely assessed [8]. The advent of DAA therapy has led to a reduction in $\mathrm{HCV}$-positive organ discard rate, from $28 \%$ in 2010 to $11 \%$ in 2015 for $\mathrm{HCV}$ - positive livers [9-15]. HCV-positive KT recipients who receive $\mathrm{HCV}$-positive grafts have been shown to have shorter waitlist times and improved graft survival compared to $\mathrm{HCV}$-positive recipients who receive $\mathrm{HCV}$-negative grafts $[16,17]$. The efficacy and safety of treating HCV-positive KT patients with DAA therapy have been shown in several studies [7, 12].

$\mathrm{HCV}$-positive KT for HCV-negative patients, however, has been less studied and raises ethical questions of known infection transmission, informed consent, and DAA insurance coverage posttransplant. Small trials of HCV NAT-positive KT into HCV-negative recipients, either with DAA treatment very early post-KT or prophylactic pre-KT showed $100 \%$ SVR rates $[18,19]$. One trial showed similar graft and patient survival rates for HCV antibody-positive $\mathrm{KT}$ recipients regardless of whether they received an $\mathrm{HCV}$-positive or $\mathrm{HCV}$-negative graft [20]. Despite the efficacy of HCV treatment, HCV-positive organs may not be routinely offered to patients. Little is known about provider attitudes toward HCV-positive $\mathrm{KT}$, particularly in the DAA era. Given the ongoing interest in HCV-positive KT, we examined nephrologists', transplant surgeons', and transplant advanced practice providers' beliefs on this topic.

\section{Methods}

This study enrolled nephrologists, transplant surgeons, and advanced practice providers in transplant nephrology at academic medical centers in the United States and Canada. These providers were identified through online searches of nephrology and transplant departments in North America. Scripted emails inviting potential participants to complete a survey were sent to the providers listed on the academic medical centers' websites. If potential participants did not respond to the initial email, identical second and if necessary third emails, over a period of 2 months, were sent inviting the providers to participate. If willing to participate, providers completed an electronic survey.

The survey used the web-based REDCap and was designed with the assistance of the University of Chicago Survey Lab. It consisted of 5 parts: transplant provider status, knowledge of $\mathrm{HCV}$ and transplantation, beliefs toward offering HCV NAT-positive kidney grafts to $\mathrm{HCV}$-positive and $\mathrm{HCV}$-negative patients, attitudes toward the doctor-patient relationship, and demographics, including age, gender, time spent performing research and/or clinical care, and United Network for Organ Sharing (UNOS) practice region. Unless otherwise stated, for the remainder of the manuscript, "HCV-positive" denotes HCV NAT positivity.

Example survey questions and statements include the following, with answer choices in parentheses:

- What do you expect the rate of cure to be of HCV for posttransplant patients who acquire active HCV (antibody positive, NAT positive) through the receipt of an infected organ? (much 
lower than the general rate; somewhat lower than the general rate; about the same as the general rate; somewhat better than the general rate; much better than the general rate)

- Does a donor with active HCV (positive antibody and viral load) likely reduce, not affect, or improve the odds of a successful transplant (at 1-year posttransplant), all other factors equal? (reduces likelihood of success; no effect on likelihood of success; improves likelihood of success)

- Despite obtaining prior written informed consent, do you worry HCV might not be curable after transplant if offering patients without $\mathrm{HCV}$ an active $\mathrm{HCV}$ organ? (not a worry; small worry; moderate worry; agree; large worry)

The entire survey can be found in online supplement 1 (see www.karger.com/doi/10.1159/000502049). Providers received a USD 10 Amazon Gift Card for their participation. The study was approved by the Institutional Review Board at the University of Chicago.

Descriptive analysis including mean and median for continuous variables and frequencies for categorical variables was performed. Student $t$ test and one-way analysis of variance (continuous data) and Fisher's Exact test (categorical data) were used to compare differences between groups.

\section{Results}

\section{Demographics}

The survey was sent to 700 providers; 99 providers (65 males, 34 females) responded (14.1\% participation rate) and were included in the study (Table 1). The median age was 44 years (range 32-73). The majority (90.9\%) were nephrologists with $62.6 \%$ of total providers transplant nephrologists, and $7.1 \%$ transplant surgeons. The majority of providers exclusively cared for adults (90.9\%) and reported being primarily clinicians (59.6\%). The median years spent in practice were 15 (range 1-41).

\section{Attitudes toward HCV-Positive KT}

All of the surveyed providers reported that $\mathrm{HCV}$ was a curable disease. Table 1 shows provider attitudes toward the use and success of HCV-positive organs. Three quarters $(74.7 \%)$ of participants believed that the cure rate posttransplant of HCV acquired from an infected transplanted organ was about the same as the general rate. Most (59.6\%) respondents thought that a $\mathrm{HCV}$-positive graft had no effect on the odds of a successful KT 1-year posttransplant, with $32.3 \%$ of providers thinking that a HCV-positive graft reduced transplant success at 1-year posttransplant. Nearly half (47.5\%) of providers thought that the use of HCV-positive kidney grafts should be for routine care only, while $38.4 \%$ believed it should be reserved for research purposes only; of the 14 providers who chose "other," 5 wrote that HCV-positive KT should be performed for both routine care and research purposes.

Compared to providers who believed the HCV cure rate was lower than the general rate, providers who believed that the HCV cure rate posttransplant was similar were significantly more likely to believe that HCV had no effect on the 1-year success of KT (68.9 vs. $22.7 \%$ respectively; $p<0.005)$. They were also significantly more likely to believe that HCV-positive KT should be used for routine care as opposed to research purposes only (55.4 vs. $27.3 \%$, respectively; $p=0.03$ ).

\section{Attitudes toward Organ Donor Sources}

The survey asked about attitudes concerning other types of organ donors. Donor organs from those who were obese, $>50$ years old, or had died from a cardiac arrest were thought to significantly reduce the likelihood of success 1-year post KT compared with a HCV-positive organ ( $p<0.005$ in all cases, Fig. 1). Grafts from a donor $<50$ years old who died accidentally were thought to significantly improve the likelihood of success 1 -year posttransplant compared with a $\mathrm{HCV}$-positive organ $(p<$ 0.005, Fig. 1).

\section{Attitudes toward Scenarios Involving HCV-Positive and HCV-Negative Kidney Grafts}

Providers were asked whether or not they would offer a $\mathrm{HCV}$-positive kidney to both $\mathrm{HCV}$-positive and HCVnegative patients in 5 different clinical contexts: (1) patient who is pre-dialysis with a glomerular filtration rate $<20 \mathrm{~mL} / \mathrm{min} / 1.73 \mathrm{~m}^{2}$; (2) patient who has been on dialysis for a year with an expected 5 year waitlist time before KT and is happy with their quality of life; (3) similar patient on HD but is unhappy with their quality of life; (4) patient who has been on HD for 4 years and is running out of HD access options; and (5) patient who is $>65$ years old with diabetes mellitus and coronary artery disease who has recently initiated dialysis. Providers were significantly more likely to offer a HCV-positive organ to $\mathrm{HCV}$-positive patients compared to HCV-negative patients in all queried circumstances ( $p<0.005$ in all cases), especially with increasing impact on patient's quality of life.

\section{Attitudes toward HCV-Positive KT and Waitlist Time}

About two-fifths (39.4\%) of participants reported that they would offer a HCV-positive organ to a HCV-negative patient if it reduced their waitlist time by 1 year. This percentage increased for every year reduction in waitlist time, with $91.9 \%$ of respondents reporting they would of- 
Table 1. Demographic, practice, and attitudes toward HCV-related kidney transplantation data among all participants

\begin{tabular}{|c|c|}
\hline & All respondents $(n=99)$ \\
\hline \multicolumn{2}{|l|}{ Demographic variables } \\
\hline Age, years, mean, median (range) & $46.4,44.0(32-73)$ \\
\hline Gender, female, $n(\%)$ & $34(34.3)$ \\
\hline \multicolumn{2}{|l|}{ Provider, $n(\%)$} \\
\hline Transplant surgeon & $7(7.1)$ \\
\hline Transplant nephrologist & $62(62.6)$ \\
\hline Nontransplant nephrologist & $28(28.3)$ \\
\hline Mid-level provider & $2(2.0)$ \\
\hline \multicolumn{2}{|l|}{ Type of practice, $n(\%)$} \\
\hline Adult & $90(90.9)$ \\
\hline Pediatrics & $1(1.0)$ \\
\hline Adult and pediatrics & $8(8.1)$ \\
\hline Years in practice, mean, median (range) & $16.1,15.0(1-41)$ \\
\hline \multicolumn{2}{|l|}{ UNOS Region, $n(\%)$} \\
\hline 1 & $6(6.2)$ \\
\hline 2 & $5(5.2)$ \\
\hline 3 & $15(15.5)$ \\
\hline 4 & $1(1.0)$ \\
\hline 5 & $17(17.5)$ \\
\hline 6 & $12(12.4)$ \\
\hline 7 & $6(6.2)$ \\
\hline 8 & $7(7.2)$ \\
\hline 9 & $11(11.3)$ \\
\hline 10 & $4(4.1)$ \\
\hline 11 & $14(14.4)$ \\
\hline \multicolumn{2}{|l|}{ Provider attitudes toward $H C V$-positive $K T$} \\
\hline HCV is a curable disease, $n(\%)$ & $99(100)$ \\
\hline \multicolumn{2}{|c|}{$\mathrm{HCV}$ cure rate of KT recipient who acquires $\mathrm{HCV}$} \\
\hline \multicolumn{2}{|l|}{ NAT + donor organ, $n(\%)$} \\
\hline Lower than the general rate & $22(22.3)$ \\
\hline About the same as the general rate & $74(74.7)$ \\
\hline Better than the general rate & $3(3.0)$ \\
\hline \multicolumn{2}{|c|}{$\mathrm{HCV}+$ transplantation for which purposes, $n(\%)$} \\
\hline Routine care only & $47(47.5)$ \\
\hline Research purposes only & $38(38.4)$ \\
\hline Other & $14(14.1)$ \\
\hline \multicolumn{2}{|c|}{$\begin{array}{l}\text { HCV-infected organ affecting the odds of a } \\
\quad \text { successful transplant at } 1 \text {-year post KT, } n(\%)\end{array}$} \\
\hline Reducing likelihood & $32(32.3)$ \\
\hline No effect & $59(59.6)$ \\
\hline Improving likelihood & $8(8.1)$ \\
\hline \multicolumn{2}{|c|}{$\begin{array}{l}\text { Would offer HCV-infected organ to a patient } \\
\quad \text { without HCV if reduced waitlist time by, year, } n \text { (\%) }\end{array}$} \\
\hline 1 & $39(39.4)$ \\
\hline 2 & $61(61.6)$ \\
\hline 3 & $82(82.8)$ \\
\hline 4 & 91 (91.9) \\
\hline
\end{tabular}

HCV, hepatitis C virus; KT, kidney transplantation; NAT, nucleic acid testing. 
Fig. 1. Providers' attitudes toward donor sources and/or donor characteristics improving, reducing, or having no effect on transplant success within 1 year after transplant. Asterisks indicate answers that the literature primarily supports. HCV, hepatitis $\mathrm{C}$ virus; IVDU, intravenous drug user.

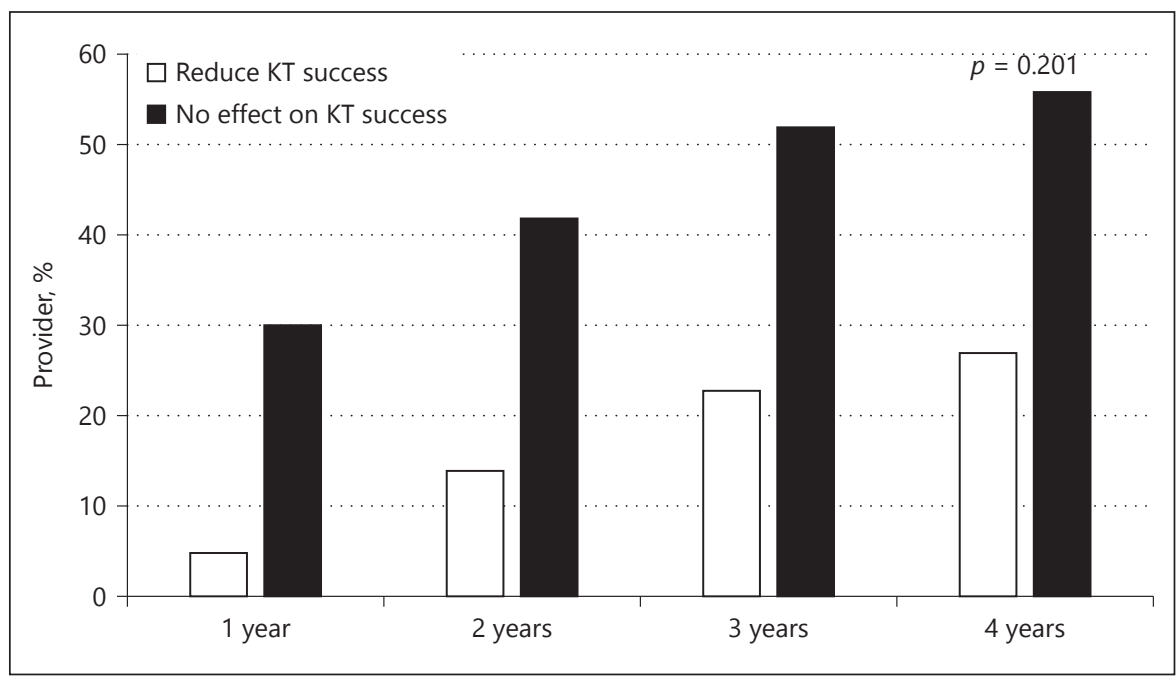

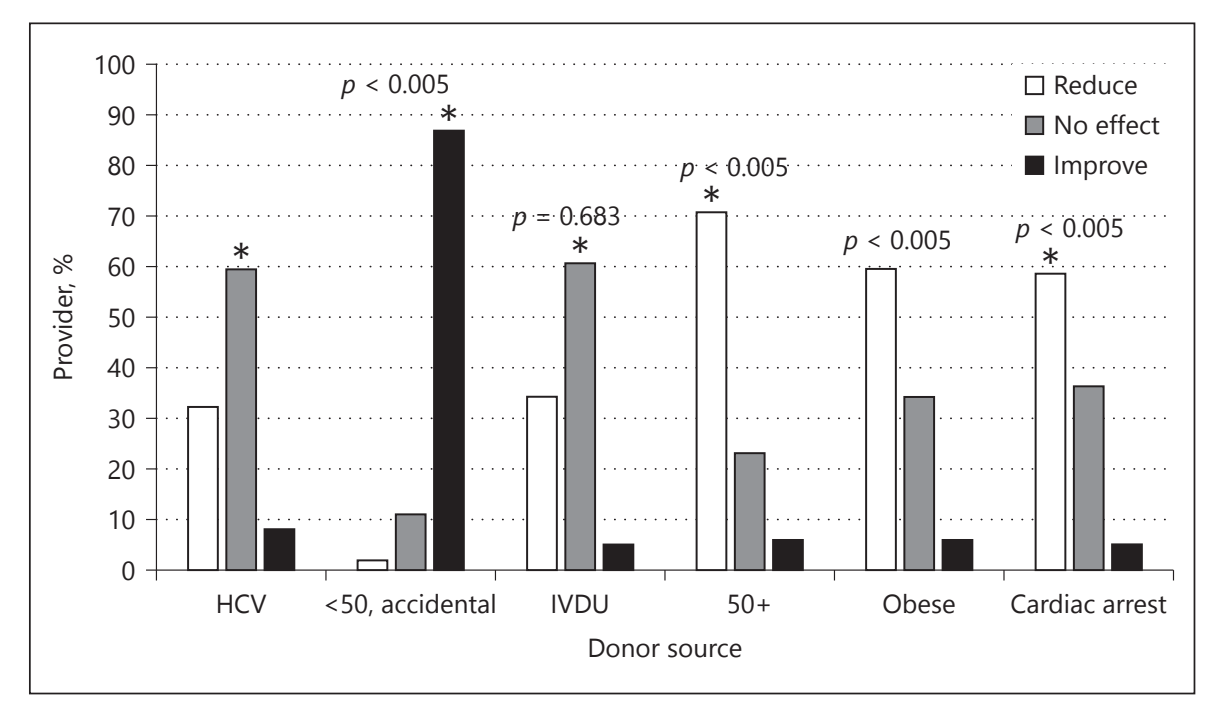

Fig. 2. Increasing willingness of providers to offer HCV NAT-positive KT to HCVnegative patients based upon waitlist reduction time. The providers are stratified according to their beliefs regarding $\mathrm{HCV}$ NAT-positive KT on transplant success. KT, kidney transplant. fer $\mathrm{HCV}$-positive $\mathrm{KT}$ to $\mathrm{HCV}$-negative patients if it reduced waitlist time by 4 years (Fig. 2). Providers who believed that HCV-positive KT had no effect on transplant success were not more likely to offer a HCV-positive organ if waitlist time decreased compared to providers who believed that $\mathrm{HCV}$-positive KT reduced transplant success $(p=0.201)$.

\section{Concerns Associated with HCV-Positive KT}

The most frequently cited concern among providers regarding $\mathrm{HCV}$-positive $\mathrm{KT}$ for $\mathrm{HCV}$-negative recipients was that HCV may not be curable after transplant, with only $14.1 \%$ of providers not worried about this possibility. $62.6 \%$ of providers reported worrying about patients' feeling "soiled" by receiving a diseased, although curable, organ, $62.6 \%$ were concerned about violating the "do no harm" medical ethic, and 59.6\% were worried about the risk of a malpractice lawsuit.

When asked whether they viewed their role as a provider primarily in terms of a medical expert, who provides the best course of treatment according to their training and science, or primarily in terms of a collaborator, who works with a patient to devise plans that take into account patient preferences, beliefs, and practical constraints, even when these run counter to science of best medical practice, $28.3 \%$ of providers viewed themselves in the former category while $71.7 \%$ classified themselves as the latter. When these 2 groups were compared, there were no significant differences when asked about provider responsibility toward a patient refusing treatment that will 
reduce morbidity and mortality as well as refusing a HCV-positive graft if they were HCV negative $(p=0.548$, $p=0.208$, respectively). There were no significant differences between transplant and nontransplant nephrologists in any queried attitudes toward HCV-positive organ transplantation.

\section{Discussion}

To the best of our knowledge, this is the first study to assess providers' attitudes specifically toward $\mathrm{HCV}$ NAT-positive KT for $\mathrm{HCV}$-negative patients in the DAA era. Although $91.9 \%$ of providers were willing to offer a $\mathrm{HCV}$-positive kidney to a $\mathrm{HCV}$-negative patient if doing so reduced waitlist time by 4 years, less than half of providers believed in offering $\mathrm{HCV}$-positive $\mathrm{KT}$ for routine care rather than for research. Approximately one-third of providers thought that a HCV-positive organ reduced transplant success at 1-year posttransplant, and the surveyed providers were more willing to offer $\mathrm{HCV}$-positive kidney grafts to $\mathrm{HCV}$-positive patients compared to HCV-negative patients. This reinforces the need for further data regarding the efficacy and safety of HCV NATpositive kidney transplantation in the DAA era and highlights the need for increased efforts at provider education.

Utilization rates for $\mathrm{HCV}$-positive kidney grafts in the pre- and post-DAA eras have been consistently shown to be lower than HCV-negative kidney grafts. From 1995 to 2009 , HCV antibody-positive kidney grafts were 2.6 times more likely to be discarded compared to HCV-negative kidney grafts for HCV-positive patients [21]. In a retrospective analysis of more than 9,000 patients who had $\mathrm{HCV}$ antibody and NAT data, $\mathrm{HCV}$ antibody-positive or NAT-positive kidney grafts were significantly less likely to be transplanted than their negative counterparts due to their HCV positivity [22]. Slightly less than half (47.5\%) of respondents believed that HCV-positive KT should be performed as routine care, while $38.4 \%$ of providers reported that this practice should be limited to research purposes only. Given that the majority of providers believed that that HCV was highly curable post-KT, perhaps rather than doubting DAA effectiveness post-KT, providers would prefer to see more efficacy and safety data before offering $\mathrm{HCV}$-positive KT routinely. Performing high-quality trials comparing outcomes for donor $\mathrm{HCV}$ NAT positive/recipient HCV NAT negative (D+/R-) KT to HCV NAT $\mathrm{D}+/ \mathrm{R}+, \mathrm{D}-/ \mathrm{R}+$, and $\mathrm{D}-/ \mathrm{R}-\mathrm{KT}$ should be a priority for the transplant community.

Provider Attitudes toward the Use of HCV-Positive Organs
A majority of providers in our study believed that the $\mathrm{HCV}$ cure rate post $\mathrm{KT}$ from a $\mathrm{HCV}$-positive donor was about the same as the general rate $(74.7 \%)$ and that a $\mathrm{HCV}$-infected organ had no effect on the odds of a success transplant at 1 -year posttransplant (59.6\%). These beliefs are likely secondary to the documented high SVR rates for KT patients in the DAA era - both for KT recipients with chronic HCV infection and for KT recipients who acquired acute $\mathrm{HCV}$ from a $\mathrm{HCV}$-positive kidney $[7,18,19]$. In addition, a 2017 study reported that $\mathrm{KT}$ recipients who received $\mathrm{HCV}$ antibody-positive kidney grafts had the same graft and survival rates as those who received HCV-negative kidney grafts; of note, only $29.6 \%$ of $\mathrm{KT}$ recipients who received $\mathrm{HCV}$ positive kidney grafts were $\mathrm{HCV}$-positive before $\mathrm{KT}$ [20].

Some providers, however, reported that the $\mathrm{HCV}$ cure rate posttransplant was less than the average rate (22.3\%). Those who believed this were significantly more likely to believe that a HCV-positive organ reduced the odds of a successful transplant at 1-year posttransplant compared to those who believed the cure rate was similar to the general rate. These differences in provider attitudes may suggest that patients may receive varying information on posttransplant outcomes as well as discrepant offers for HCV-positive grafts, depending on provider beliefs.

While initial data are positive regarding efficacious SVR rates and outcomes for HCV-positive KT recipients - both who acquired HCV before or from KT - few clinical trials have been conducted specifically examining these issues. Two open label, nonrandomized pilot trials, each with ten HCV-negative patients who underwent HCV NAT-positive KT, demonstrated $100 \%$ SVR rates $[18,19]$. These trials have led to a surge in interest in HCV $\mathrm{D}+/ \mathrm{R}-\mathrm{KT}$ and may herald a paradigm shift in transplant medicine. Given the low utilization rates of $\mathrm{HCV}$-positive kidney grafts, increased provider education on the efficacious HCV SVR rates and preliminary outcome and safety data may lead to increased use of these grafts.

Little data exist regarding provider attitudes toward Public Health Service (PHS) increased risk (IR) organ donation, including for HCV-positive KT, both into HCVpositive and HCV-negative patients. From 2005 to 2008, PHS IR kidney grafts were $8.2 \%$ less likely, compared to non-PHS IR kidneys, to be used for KT, even though posttransplant survival rates were equivalent between the 2 groups at 2-year follow-up [23]. Similarly, a study examining patients from 2010 to 2013 showed that PHS IR kidney grafts were used at a lower rate than non-PHS IR 
kidneys (76.7 and 83.7\%, respectively) [24]. A 2014 survey of 87 nonphysician transplant providers, $64 \%$ of whom were registered nurses, found that $56 \%$ of respondents felt uncomfortable discussing informed consent for PHS IR kidney grafts [25]. In this study, $99 \%$ of those surveyed also wanted supplemental education on PHS IR kidney donation.

Although some respondents expressed concerns about HCV positivity on transplant success, the participants were more likely to view obesity, a kidney from a donor $>50$ years old, or a kidney from a donor who died of cardiac arrest as more likely to reduce posttransplant success than a HCV-positive kidney graft. A potential explanation for these differences includes the previously discussed equivalent patient and graft survival rates as well high SVR rates for $\mathrm{HCV}$-positive $\mathrm{KT}$ recipients in the DAA era. Other reasons may be that HCV-positive donors have been shown to be younger and have less comorbidities than non HCV-positive donors [20, 26-28]. In addition, kidney grafts from those who are older and who have died from cardiac arrest have been shown to lead to worse posttransplant outcomes [29, 30]. Intravenous drug user donor grafts have been shown to have equivalent outcomes to nonintravenous drug user grafts, and controversy exists regarding whether donor obesity affects KT outcomes positively or negatively [31,32].

Providers were also more likely to offer $\mathrm{HCV} \mathrm{D+/R+}$ $\mathrm{KT}$ compared to HCV D+/R- KT. This belief coincides with the most frequently cited provider concern: HCV curability, with $85.9 \%$ citing this as a worry they had regarding $\mathrm{HCV}$-positive KT. Although the vast majority of providers had this concern, $79.8 \%$ of providers reported that the general cure rate of $\mathrm{HCV}$ was greater than $90 \%$, and approximately 3 -quarters of them believed that the $\mathrm{HCV}$ cure rate post-KT was the same as the general rate. In addition to rare SVR failures, obtaining insurance approval for these sometimes costly DAA therapies could be potential reasons for this concern $[20,33]$.

There are several limitations to our study. The response rate was $14.1 \%$, resulting in potential selection bias. Because the survey lacked questions that identified individuals, it is impossible to compare participants' and nonparticipants' demographic information, gender, profession, and UNOS region, which may have aided in results analysis and mitigated potential downsides to the response rate. Future studies on provider attitudes should link participants' identity to their responses in order to analyze these potential differences. The online survey format may have contributed to the response rate seen as multiple studies have documented a lower response rate for online surveys of $17.9-22 \%$, including ongoing declines in online survey participation each year, compared to traditional written surveys [34-36]. Another limitation is the paucity of transplant surgeon and mid-level provider representation in our study. Further studies analyzing these providers' attitudes - particularly transplant surgeons - are necessary given the essential role they play in KT care and the transplant consent process. Generalizability of our results, both across the United States as well as worldwide, may be a limitation. All UNOS regions are represented in our survey; however, given the number of participants, 6 regions each have less than ten percent of those surveyed. In addition, overseas provider attitudes of $\mathrm{HCV}$-positive $\mathrm{KT}$ may differ, given the divergent incidence of $\mathrm{HCV}$ in different countries and the known varying cultural beliefs toward $\mathrm{HCV}$ in general [37-39].

In addition, although this study reports providers' beliefs concerning HCV-positive KT, these attitudes are not coupled with providers' actions or patient beliefs. Although outside the scope of this survey study, a robust study linking attitudes and clinical practice would shed light on if provider beliefs toward HCV-positive KT are influencing their clinical practice. A 2019 study examining solid organ (primarily kidney) transplant candidates' attitudes toward HCV NAT-positive KT showed that $46 \%$ of patient were willing to receive a HCV NAT-positive kidney, $30 \%$ were unsure, and $24 \%$ were unwilling [40]. The candidates amenable to HCV NAT-positive KT had significantly higher levels of trust in their physicians [40]. These data reinforce the need for greater provider and patient education toward HCV NAT-positive KT.

In summary, less than half of providers surveyed reported that $\mathrm{HCV}$-positive KT should be performed for clinical purposes only. However, a majority of providers in our study believed that the cure rate posttransplant of $\mathrm{HCV}$ acquired from an infected transplanted organ was about the same as the general rate and that a HCV-infected organ had no effect on the odds of a successful transplant at 1-year posttransplant. HCV-positive KT was more appealing to providers than grafts from obese donors, those $>50$ years old, and donors who died from cardiac arrest. Increasing waitlist times were also associated with increased provider willingness to offer HCV-positive KT. More research on the safety and efficacy, as well as standardization of protocols, of DAA therapy post-KT will likely influence providers' beliefs toward HCV-positive KT. Provider education regarding the high HCV cure rates post-KT may result in an increase in routine $\mathrm{HCV}$ positive KT. 


\section{Acknowledgments}

The authors would like to acknowledge all the participating providers who participated in the survey.

\section{Statement of Ethics}

Subjects have given their written informed consent. The study protocol has been approved by the University of Chicago Medical Center's committee on human research. No animal experiments were performed in the course of this research.

\section{Disclosure Statement}

The authors have no conflicts of interest to declare.

\section{Funding Sources}

R.C. is funded by NIH DK078772. S.P. received funding from the University of Chicago Biological Science Division's Office of Diversity and Inclusion and The Gastroenterology Research Fund Associates Board.

\section{Author Contributions}

T.C.: acquisition of data; interpretation of data; drafting of manuscript. J.K., K.S., and A.N.: acquisition of data. H.Y.: critical revision of manuscript for important intellectual content. R.C.: study concept and design; critical revision of manuscript for important intellectual content. S.P.: study concept and design; interpretation of data; drafting of manuscript; critical revision of manuscript for important intellectual content.

\section{References}

1 Shafran D, Kodish E, Tzakis A. Organ shortage: the greatest challenge facing transplant medicine. World J Surg. 2014 Jul;38(7):16507.

2 Hart A, Smith JM, Skeans MA, Gustafson SK, Wilk AR, Robinson A, et al. OPTN/SRTR 2016 Annual Data Report: kidney. Am J Transplant. 2018 Jan;18(suppl 1):18-113.

3 Armstrong GL, Wasley A, Simard EP, McQuillan GM, Kuhnert WL, Alter MJ. The prevalence of hepatitis $C$ virus infection in the United States, 1999 through 2002. Ann Intern Med. 2006 May;144(10):705-14.

4 Baid-Agrawal S, Pascual M, Moradpour D, Somasundaram R, Muche M. Hepatitis C virus infection and kidney transplantation in 2014: what's new? Am J Transplant. 2014 Oct; 14(10):2206-20.

5 Ellingson K, Seem D, Nowicki M, Strong DM, Kuehnert MJ; Organ Procurement Organization Nucleic Acid Testing Yield Project Team. Estimated risk of human immunodeficiency virus and hepatitis $\mathrm{C}$ virus infection among potential organ donors from 17 organ procurement organizations in the United States. Am J Transplant. 2011 Jun;11(6):1201-8.

6 Rossaro L, Torruellas C, Dhaliwal S, Botros J, Clark G, Li CS, et al. Clinical outcomes of hepatitis $\mathrm{C}$ treated with pegylated interferon and ribavirin via telemedicine consultation in Northern California. Dig Dis Sci. 2013 Dec; 58(12):3620-5.

7 Lin MV, Sise ME, Pavlakis M, Amundsen BM, Chute D, Rutherford AE, et al. Efficacy and safety of direct acting antivirals in kidney transplant recipients with chronic hepatitis C virus infection. PLoS One. 2016 Jul;11(7): e0158431.

8 Reese PP, Abt PL, Blumberg EA, Goldberg DS. Transplanting Hepatitis C-Positive Kidneys. N Engl J Med. 2015 Jul;373(4):303-5.
9 Afdhal N, Reddy KR, Nelson DR, Lawitz E, Gordon SC, Schiff E, et al.; ION-2 Investigators. Ledipasvir and sofosbuvir for previously treated HCV genotype 1 infection. N Engl J Med. 2014 Apr;370(16):1483-93.

10 Afdhal N, Zeuzem S, Kwo P, Chojkier M, Gitlin N, Puoti M, et al.; ION-1 Investigators. Ledipasvir and sofosbuvir for untreated $\mathrm{HCV}$ genotype 1 infection. N Engl J Med. 2014 May;370(20):1889-98.

11 Bowring MG, Kucirka LM, Massie AB, Luo X, Cameron A, Sulkowski M, et al. Changes in Utilization and Discard of Hepatitis C-Infected Donor Livers in the Recent Era. Am J Transplant. 2017 Feb;17(2):519-27.

12 Eisenberger U, Guberina H, Willuweit K, Bienholz A, Kribben A, Gerken G, et al. Successful Treatment of Chronic Hepatitis C Virus Infection With Sofosbuvir and Ledipasvir in Renal Transplant Recipients [Internet]. Transplantation. 2017 May; 101(5): 980-6.

13 Forns X, Lee SS, Valdes J, Lens S, Ghalib R, Aguilar $\mathrm{H}$, et al. Glecaprevir plus pibrentasvir for chronic hepatitis $\mathrm{C}$ virus genotype $1,2,4$, 5 , or 6 infection in adults with compensated cirrhosis (EXPEDITION-1): a single-arm, open-label, multicentre phase 3 trial. Lancet Infect Dis. 2017 Oct;17(10):1062-8.

14 Gane E, Lawitz E, Pugatch D, Papatheodoridis G, Bräu N, Brown A, et al. Glecaprevir and Pibrentasvir in Patients with HCV and Severe Renal Impairment. N Engl J Med. 2017 Oct; 377(15):1448-55.

15 Reddy KR, Everson GT, Flamm SL, Denning JM, Arterburn S, Brandt-Sarif T, et al. Ledipasvir/sofosbuvir with ribavirin for the treatment of $\mathrm{HCV}$ in patients with post transplant recurrence: preliminary results of a prospective, multicenter study. Presented at: 65th Annual Meeting of the American
Association for the Study of Liver Diseases; November 7-11, 2014; Boston, MA. Abstract 8.

16 Patwardhan VR, Curry MP. Reappraisal of the hepatitis $C$ virus-positive donor in solid organ transplantation. Curr Opin Organ Transplant. 2015 Jun;20(3):267-75.

17 Scalea JR, Barth RN, Munivenkatappa R, Philosophe B, Cooper M, Whitlow V, et al. Shorter waitlist times and improved graft survivals are observed in patients who accept hepatitis $\mathrm{C}$ virus+ renal allografts. Transplantation. 2015 Jun;99(6):1192-6.

18 Durand CM, Bowring MG, Brown DM, Chattergoon MA, Massaccesi G, Bair N, et al. Direct-acting antiviral prophylaxis in kidney transplantation from hepatitis $C$ virus-infected donors to noninfected recipients an openlabel nonrandomized trial. Ann zof. Intern Med. 2018;168(8):523-40.

19 Goldberg DS, Abt PL, Blumberg EA, Van Deerlin VM, Levine M, Reddy KR, et al. Trial of Transplantation of HCV-Infected Kidneys into Uninfected Recipients. N Engl J Med. 2017 Jun;376(24):2394-5.

20 Trotter PB, Summers DM, Ushiro-Lumb I, Robb M, Bradley JA, Powell J, et al. Use of organs from hepatitis $\mathrm{C}$ virus-positive donors for uninfected recipients: A Potential CostEffective Approach to Save Lives? Transplantation. 2018 Apr;102(4):664-72.

21 Kucirka LM, Singer AL, Ros RL, Montgomery RA, Dagher NN, Segev DL. Underutilization of hepatitis C-positive kidneys for hepatitis Cpositive recipients. Am J Transplant. 2010 May;10(5):1238-46.

22 Kling CE, Perkins JD, Landis CS, Limaye AP, Sibulesky L. Utilization of Organs From Donors According to Hepatitis C Antibody and Nucleic Acid Testing Status: time for Change. Am J Transplant. 2017 Nov;17(11):2863-8. 
23 Duan KI, Englesbe MJ, Volk ML. Centers for Disease Control 'high-risk' donors and kidney utilization. Am J Transplant. 2010 Feb; 10(2):416-20.

24 Volk ML, Wilk AR, Wolfe C, Kaul DR. The "PHS Increased Risk" Label Is Associated With Nonutilization of Hundreds of Organs per Year. Transplantation. 2017 Jul;101(7): 1666-9.

25 Gordon EJ, Mullee J, Beauvais N, Warren E, Theodoropoulos N, McNatt G, et al.; Increased Risk Organ and Informed Consent (IROIC) Workgroup. Education and informed consent about increased risk donor kidneys: a national survey of non-physician transplant providers. Transpl Infect Dis. 2014 Apr;16(2):251-60.

26 Fishman JA, Forns X. HCV-Positive Donor Organs in Solid Organ Transplantation: "Mind the Gap!". Am J Transplant. 2017 Nov; 17(11):2755-6.

27 Fleetwood VA, Lusciks J, Poirier J, Hertl M, Chan EY. Utilization of Public Health Service Increased Risk Donors Yields Equivalent Outcomes in Liver Transplantation. J Transplant. 2016;2016:9658904.

28 Pruett TL, Clark MA, Taranto SE. Deceased Organ Donors and PHS Risk Identification:
Impact on Organ Usage and Outcomes. Transplantation. 2017 Jul;101(7):1670-8.

29 Huang E, Segev DL, Rabb H. Kidney transplantation in the elderly. Semin Nephrol. 2009 Nov;29(6):621-35.

30 Legendre C, Canaud G, Martinez F. Factors influencing long-term outcome after kidney transplantation. Transpl Int. 2014 Jan;27(1): 19-27.

31 Lin SJ, Koford JK, Baird BC, Hurdle JF, Krikov S, Habib AN, et al. Effect of donors' intravenous drug use, cigarette smoking, and alcohol dependence on kidney transplant outcome. Transplantation. 2005 Aug;80(4):4826.

32 Buggs J, Mehta R, Boone J, Adesunkanmi M, Rogers E, Kumar A, et al. Outcomes of Donor and Recipient Obesity in Kidney Transplantation. Am Surg. 2018 Sep;84(9):e375-7.

33 Kling CE, Limaye AP, Landis CS, Sibulesky L. Expanding access to transplantation with hepatitis C-positive donors: A new perspective on an old issue. Clin Transplant. 2017 Feb;31(2):2016-7.

34 Aerny-Perreten N, Domínguez-Berjón MF, Esteban-Vasallo MD, García-Riolobos C. Participation and factors associated with late or non-response to an online survey in pri- mary care. J Eval Clin Pract. 2015 Aug;21(4): 688-93.

35 Kongsved SM, Basnov M, Holm-Christensen $\mathrm{K}$, Hjollund NH. Response rate and completeness of questionnaires: a randomized study of Internet versus paper-and-pencil versions. J Med Internet Res. 2007 Sep;9(3): e25.

36 van Gelder MM, Bretveld RW, Roeleveld N. Web-based questionnaires: the future in epidemiology? Am J Epidemiol. 2010 Dec; 172(11):1292-8.

37 Younossi ZM. Hepatitis C Infection: A Systemic Disease. Clin Liver Dis. 2017 Aug;21(3): 449-53.

38 Alizadeh AHM, Ranjbar M, Yadollahzadeh M. Patient concerns regarding chronic hepatitis B and C infection. East Mediterr Health J. 2008 Sep-Oct;14(5):1142-7.

39 Minuk GY, Gutkin A, Wong SG, Kaita KD. Patient concerns regarding chronic hepatitis C infections. J Viral Hepat. 2005 Jan;12(1): 51-7.

40 Couri T, Cotter TG, Chen D, Hammes M, Reddy B, Josephson M, et al. Use of Hepatitis C Positive Organs: Patient Attitudes in Urban Chicago. Am J Nephrol. 2019;49(1):3240 . 